\title{
Infecciones nosocomiales: tendencias seculares de un programa de control en México
}

\author{
Samuel Ponce de León, M.C., M en C., ${ }^{(1)}$ M. Sigfrido Rangel-Frausto, M.C., M. en C., ${ }^{(2)}$ \\ Josué I. Elías-López, M.C..(1) $C$ armen Romero-O liveros, Lic. en Enf., ${ }^{(2)}$ \\ Martha Huertas-Jiménez, Lic. en Enf. ${ }^{(2)}$
}

\section{Ponce de León S, Rangel-Frausto MS, Elías-López JI, Romero- OliverosC, Huertas-Jiménez M. Infeccionesnosocomiales: tendenciasseculares de un programa de control en México. Salud Publica Mex 1999;41 suppl 1:S5-S11.}

\section{Resumen}

Objetivo. Determinar la frecuencia de infecciones nosocomiales en el Instituto $\mathrm{N}$ acional de la N utrición Salvador Zubirán (IN N SZ) desde la instauración del programa de vigilancia hasta la actualidad y co nocer su impacto en estancia hospitalaria y mor talidad. Material y métodos. Se realizó un estudio descriptivo, retrospectivo y retrolectivo en un hospital de referencia de tercer nivel de atención. Se obtuvieron las frecuencias, el tipo y la distribución de infecciones nosocomiales de la base de datos del D epartamento de Epidemiología Hospitalaria entre 1991 y 1996. Se determinó en cada paciente el grado de gravedad de su enfermedad mediante la escala de McC abe-Jackson, así como la edad promedio y el tiempo de estancia hospitaria. Se aplicó la prueba de $c^{2}$ para tendencias de los diferentes parámetros evaluados. El estudio se dividió en tres etapas: prerremodelación (1991-1993), remodelación (1994-1995) y posremodelación (1996). Se compararon los periodos prerremodelación contra posremo delación. Resultados. Durante este periodo (1991-1996) la tasa de infecciones nosocomiales fue de 8.6 por 100 egresosen promedio, con una disminución de $20 \%$ en su frecuenciaalo largo del mismo $(p<0.01)$, comparando las tasas que se observaron durante la prerremodelación con las de la posrremodelación. El área hospitalaria con mayor frecuenciade infecciones es la Unidad deTerapia Intensiva (26.9 infecciones por 100 egresos), seguidadeáreas de hospitalización con cuartos compartidos (9.47\%) ycon cuartosprivados (7.5\%). Esitio deinfección másfrecuentefue el de vías urinarias (26.6\% del total de episodios), seguido por infección deheridaquirúrgica (24.4\%), neumoníanosocomial (12.1\%) ybacteriemia primaria(9.5\%). Seobservó unadisminución estadísticamentesignificativa
Ponce de León S, Rangel-Frausto MS, Elías-López JI, RomeroOliverosC, Huertas-Jiménez $M$.

Nosocomial infections: Secular trends

of a control program in Mexico.

Salud Publica Mex 1999;41 suppl 1:S5-S11.

\begin{abstract}
A bstract
Objective.To know the trend of nosocomial infections rates at a third level hospital since the begining of the infection control program until the present and to evaluate the impact in mortality and length of hospital stay. Material and methods A study descriptive, retrospective and retrolective was done in a reference hospital providing tertiary care in Mexico City. Recorded data included rates, type of nosocomial infections and distribution of nosocomial infections from the database of H ospital Epidemiology D ivision between 1991 to 1996. In every patient the degree of severity diseasewas evaluated accordingto the McC abe-Jackson's scale, we also collected age and the length of stay.Statistical analysis was done with $c^{2}$ test for tendencies of different evaluated parameters.This study was divided in three periods: before remodel (1991-1993), to remodel (1994-1995) and after remodel (1996). Compared the before remo del period against after remodel period. Results. In thestudy period, mean noso comial infections ratio was of 8.6 by 100 discharges, showingadecrease of $20 \%$ ( $p<$ 0.01 ). Hospital area with the highest infections rate was IC U (26.9 by 100 discharge), followed by general ward of hospitalization with shared rooms (9.47) and private rooms (7.5). Urinary tract infections was the most frequent (26.6\%), followed by surgical wound infection (24.4\%), pneumonia (12.1\%) and primary bacteremia (9.5\%). Rates of urinary tract infections and primary bacteremias had decreased significantly $(p<$ 0.05 and $p<0.00001$ respectively), while surgical wound infections and pneumoniasincreased $(p<0.005$ and $p<0.00001$ respectively)A Associated mortality diminished in $36 \%$ ( $p<0.00001)$.The length of stay diminished 42.8\%. Therewas not differences in the severity of disease alongthis period. Condusions. Since the establishment of the nosocomial infections survillance and control program at the IN N SZ on 1985,
\end{abstract}

(1) Jefe de la División de Epidemiología Hospitalariay Control de Calidad de laAtención Médica,Instituto N acional de la N utrición Salvador Zubirán (IN N SZ),México.

(2) División de Epidemiología Hospitalariay Control de Calidad de laA tención Médica,IN N SZ,México.

(3) Médico pasante en servicio social, IN N SZ, México.

Fecha de recibido: 19 de febrero de 1998 • Fecha de aprobado: 6 de agosto de 1998

Solicitud de sobretiros: Dr. Samuel Ponce de León. Instituto N acional de la N utrición Salvador Zubirán. División de Epidemiología Hospitalaria y Control de Calidad de la Atención Médica.Vasco de Q uiroga 15,Tlalpan 14000 México, D.F. 
en lafrecuencia de infecciones de vías urinarias y bacteriemias primarias ( $p<0.05$ y $p<0.00001$, respectivamente), mientras que las infecciones de herida quirúrgica y las neumonías aumentaron $(p<0.005$ y $p<$ 0.00001 , respectivamente). La mortalidad aso ciadadisminuyó $36 \%$ ( $p<$ 0.00001). Laestanciahospitalariadisminuyó en $42.8 \%$. N o se encontraron diferencias significativas en la gravedad de los enfermos. CondusonesD esde que comenzó a operar,en 1985,el programade control deinfecciones en el IN N SZ, lafrecuenciade infeccionesnosocomiales ha disminuido en $56 \%$, así como la mortalidad asociada(36\%). Estos cambios se deben tanto a dicho programa como a la reducción de la estancia hospitalaria, y no parecen estar relacionados con laedad de los pacientesni con la gravedad de su padecimiento.

Palabras clave: infección hospitalaria/tendencias; eficacia; mortalidad; México nosocomial infections rate ( $56 \%$ ) and the associated mortality (36\%) have diminished.These changes are consequence of the establishment ofa nosocomial infections control program and the decrease of length of stay and doesnot seems to be related to the age or to the severity of the disease of the hospitalized patients.

Key words: cross infection/trends; efficacy; mortality; Mexico
$\mathrm{L}$ as infecciones nosocomiales (IN) son consecuencia directa de la atención médica y se definen como aquellas que no estaban presentes o en periodo de incubación al momento en que el paciente ingresó al hospital. Se estima que de los 35 millones de pacientes que son hospitalizados en Estados Unidos de América (EUA), al menos 2.5 millones desarrollarán una infección nosocomial, es decir, habrá 5.7 infecciones por cada 100 admisiones. ${ }^{1}$ En ese país en promedio se informan incidencias de infecciones nosocomiales de 3 a $5 \%{ }^{2} \mathrm{~A}$ pesar de la magnitud del problema, hasta la fecha muchos de los países en vías de desarrollo no se han percatado de la importancia del control de las IN, y en los escasos reportes basados en sistemas de vigilancia inadecuados se informa una muy baja frecuencia. En América Latina, y pese a los esfuerzos de las naciones por enfrentar este problema, únicamente $5 \%$ de los hospitales informan tener comités con programas regulares de control de IN. ${ }^{3}$

A partir de estudios realizados en hospitales de referencia y en algunos otros de segundo nivel en México, se asume que el promedio de IN es de 10 a 15\%; esto significaría que ocurrieron aproximadamente de 600000 a 750000 casos de IN entre los 6600000 pacientes que recibieron atención médica hospitalaria en 1996. El impacto más importante de este problema es la mortalidad, la cual se estima que es de $5 \%$ en promedio, lo que significa que cada año mueren entre 30000 y 45000 pacientes con una IN asociada. ${ }^{4}$

Las características de la medicina que se practica en la actualidad se asocian a un alto riesgo de IN, como consecuencia de la realización de cirugías más extensas y complejas, de procedimientos invasivos más agre- sivos, así como de enfermedades y tratamientos inmunosupresores. ${ }^{5}$ Por otra parte, existe un mayor interés por parte del público respecto a la calidad de la atención médica, mismo que es reforzado por el manejo que hacen los medios de comunicación de este tipo de información. ${ }^{67}$

Para controlar las $I N$, ante todo es necesario conocer la magnitud del problema y sus características, y después poner en marcha las medidas necesarias para prevenirlas.

Para disponer de datos fidedignos acerca de la magnitud de este problema en un hospital, es necesario contar con un sistema de vigilancia eficaz que funcione de manera permanente y que cubra todos los servicios de hospitalización, atendiendo en particular los sitios de mayor riesgo (unidades de cuidados intensivos, servicio de hemodiálisis, etc.). ${ }^{2}$ El costo beneficio de un sistema tal, puede valorarse por medio de la frecuencia, los costos y el tiempo de hospitalización resultantes de cada episodio de IN. El costo promedio de una IN en dólares estadunidenses varía de 382 a 1833 , erogación que se evitaría por cada episodio prevenido. ${ }^{7}$ El establecimiento de programas de control y vigilancia epidemiológica en hospitales de EUA ha resultado en una disminución de hasta $32 \%$ en la frecuencia de IN en un lapso de cinco años. ${ }^{89}$ En México, Ponce de León y colaboradores analizaron los resultados que se obtuvieron al poco tiempo de haber puesto en marcha un programa de este tipo en el Instituto Nacional dela Nutrición Salvador Zubirán (INNSZ) y encontraron una reducción de $22.3 \%$ en los episodios de infección, con la consecuente disminución de la mortalidad y de los costos. ${ }^{10}$

En la mayoría de los hospitales generales la causa más frecuente de IN son las de vías urinarias, seguidas por las de herida quirúrgica, bacteriemias y neumonías. Estas infecciones se relacionan directamente con técnicas y 
procedimientos que son susceptibles de supervisión y mejoramiento, de tal manera que se pueden disminuir las tasas de infección. Otro factor que se relaciona con el desarrollo de IN es la edad del paciente -el National Nosocomial Infection Surveillance ha informado que $54 \%$ de las IN se presentaron en ancianos-; asimismo, el tiempo de estancia hospitalaria ha sido descrito como un factor determinante para el desarrollo de IN, pues a mayor estancia mayor número de IN; ${ }^{11,12}$ de la misma manera, algunos estudios demuestran que la gravedad de las enfermedades subyacentes de los pacientes que ingresan al hospital es un predictor de riesgo para adquirir una IN. ${ }^{13}$

En este trabajo se describen las tendencias que se observaron entre 1991 y 1996 en cuanto a frecuencia, agentes etiológicos y características demográficas de cifras proporcionadas por la base de datos del programa de control de infecciones nosocomiales del INNSZ.

\section{Material y métodos}

El INNSZ es un hospital de referencia del tercer nivel de atención médica; cuenta con tres pisos de hospitalización, de los cuales los dos primeros tienen cuartos que, antes de 1995, eran de seis camas y que, a partir de 1995, son sólo de cuatro. El tercer piso tiene sólo cuartos individuales. Cuenta con 160 camas censables, distribuidas de la siguiente manera: 120 en los pisos primero y segundo, 32 en los cuartos privados del tercer piso y ocho en urgencias; además, existen 14 camas en cuidados intensivos. El personal que labora en los tres pisos de hospitalización, incluyendo médicos y enfermeras, es rotatorio. El cuidado de los pacientes es responsabilidad directa de los residentes de medicina interna, con rotaciones de dos a cuatro meses, bajo la supervisión de médicos de base.

La División de Epidemiología Hospitalaria vigila la frecuencia y el tipo de IN desde 1982; cuenta con cuatro enfermeras especialmente capacitadas para esto. La sensibilidad del programa de vigilancia es de $98 \%$, y la especificidad, de $95 \% .{ }^{14}$ La vigilancia es activa y permanente e incluye todas las áreas del hospital; se revisó a todos los pacientes que ingresaron, sus expedientes, las curvas térmicas y las indicaciones médicas de antibióticos. Además, el equipo de enfermeras revisa diariamente los resultados de los cultivos en el laboratorio de microbiología e interroga a las enfermeras y médicos encargados de cada piso sobre la presencia de infecciones o fiebre.

En un formato ad hoc se lleva a cabo el registro de IN, incluyendo datos acerca del tipo de infección, la fecha, el sitio, los microrganismos identificados, los tratamientos instituidos, los diagnósticos de base y las complicaciones. Los datos se ingresan a un programa de cómputo (Paradox 5) especialmente diseñado para el mismo. Con el propósito de cubrir los objetivos del estudio, se revisaron las frecuencias, el tipo, la distribución y el impacto de las IN de 1991 a 1996.

La frecuencia de IN se calculó por número de episodios y número de pacientes infectados por mes entre el número de egresos en ese mes. Se hicieron distintos ajustes de acuerdo con el lugar (unidad de cuidados intensivos, pisos primero, segundo o tercero, etc.), por tipo de infección (bacteriemias, neumonías, infecciones de vías urinarias) y por periodo (año).

Mediante la escala McCabe-Jackson, todos los pacientes con IN se clasificaron, de acuerdo con sus diagnósticos de ingreso, en tres grupos que correspondían al riesgo de muerte: rápidamente fatales (si la sobrevida al momento del ingreso era menor a seis meses); últimamente fatales (si la sobrevida era menor a cinco años) y no fatales (si no se esperaba que fallecieran en dicho periodo). ${ }^{15}$ Lo anterior permitió conocer la gravedad de los pacientes hospitalizados en cada periodo. Además, se incluyó la edad como factor de riesgo independiente para el desarrollo de IN, para lo cual se comparó la edad de los pacientes en cada periodo. Por otra parte, también se analizó el promedio de estancia hospitalaria (antes del desarrollo de IN) en cada periodo.

Finalmente, se identificó a aquellos pacientes que adquirieron una IN y que fallecieron, sin tomar en consideración la causa directa (en cada uno de los periodos), para así determinar la mortalidad asociada a IN.

El periodo que comprendió el estudio se dividió en tres etapas: una iba de 1994 a 1995; en ella el hospital fue remodelado y ampliado en sus instalaciones. La otra fue previa a ese periodo (antes de la construcción) y abarcó los años de 1991 a 1993; la última etapa es posterior a la construcción en 1996. Se utilizaron pruebas de $c^{2}$ para identificar diferencias estadísticas en las tendencias de cada parámetro evaluado en este estudio. Se comparó el periodo previo a la construcción vs el posterior a la misma.

En este estudio retrospectivo se revisaron los archivos del sistema de vigilancia epidemiológica a cargo de la División de Epidemiología y Control de Calidad de la Atención Médica, de 1991 a 1996.

\section{Resultados}

De 1991 a 1996 en el INNSZ hubo un promedio de 3178 egresos anuales, de los cuales $8.6 \%$ tuvieron una IN (promedio de 275 pacientes por año), lo cual se traduce en una razón promedio de 11.5 infecciones por 100 egresos. Al comparar la razón de infecciones de 1991-1993 con la de 1996 se aprecia una diferencia estadísticamente significativa (c ${ }^{2} 9.1, \mathrm{RM} 1.22$, IC95\% 1.07-1.40, $p<0.01$ ) (cuadro I). 
Elárea hospitalaria donde se detectó el mayor número de IN fue la Unidad de Terapia Intensiva (UTI) con una tasa promedio de 26.9 infecciones por 100 egresos, de tal manera que superó hasta en tres veces la tasa promedio de los sectores de hospitalización (pisos primero y segundo, 9.47 infecciones por 100 egresos, y piso tercero, 7.5 infecciones por 100 egresos) y urgencias (7.2 infecciones por 100 egresos) (cuadro II). La frecuencia de infecciones en la UTI se ha mantenido sin cambios importantes, al igual que en el tercer piso de hospitalización. Los pisos primero y segundo se analizaron en conjunto por tener el mismo número de camas por cuarto y una enfermera por cada 10 pacientes, y se encontró una disminución estadísticamente significativa entre las etapas prestablecidas (1991-1993 comparado con 1996: $c^{2}$ 9.26, RM1.28,IC95\% 1.09-1.51, p<0.01). Sin embargo, en el tercer piso de hospitalización la frecuencia de infecciones nosocomiales ha permanecido constante en los últimos cinco años. La disminución más significativa se observó en el servicio de urgencias ( $c^{2} 18.6$, RM 4.81, IC95\% 2-12, p< 0.00005).

El sitio de infección más frecuente fueron las vías urinarias (26.6\% del total de episodios), seguido por infección de herida quirúrgica (24.4\%), neumonía nosocomial $(12.1 \%)$, bacteriemia primaria $(9.5 \%)$ y bacteriemia secundaria (5.1\%) (cuadro III). Al comparar la proporción de infecciones de vías urinarias entre 1991-1993 y 1996, se observó una disminución estadísticamente significativa ( $c^{2} 4.12$, RM 1.36, IC95\% 1-1.85, $p<0.05)$ al igual que para bacteriemias primarias $\left(\mathrm{c}^{2}\right.$ 19.8, RM 3.35, IC95\% 1.86-6.14, $p<0.00001)$. Al comparar los mismos periodos para infecciones de herida quirúrgica y neumonías, se encontró un aumento estadísticamente significativo ( $c^{2} 7.96$, RM 1.55, IC95\% 1.14-2.12, $p<$ 0.005, y c c $^{2} .69$, RM 2.09, IC95\% 1.17-2.94, $p<0.00001$, respectivamente).

Entre las infecciones de vías urinarias, Escherichia coli y Candida spp. constituyeron $50 \%$ del total de microrganismos aislados. De los restantes, Enterococo, Klebsiella pneumoniae y Pseudomonas aeruginosa repre-

\section{Cuadrol}

\section{TASA POR 100 EGRESOSY TASA DE INCIDENCIA DE INFECCIONES NOSOCOMIALESEN EL IN NSZ, 1991-1996}

\begin{tabular}{lrrrrrr} 
& 1991 & 1992 & 1993 & 1994 & 1995 & 1996 \\
Egresos & 2909 & 3177 & 3582 & 2438 & 3788 & 3175 \\
\hline Pacientes infectados & 259 & 284 & 325 & 216 & 320 & 246 \\
\hline Infecciones & 364 & 361 & 427 & 306 & 412 & 316 \\
\hline Tasa X 100 & 12.5 & 11.4 & 11.9 & 12.6 & 10.9 & 10.0 \\
\hline Incidencia & 8.9 & 8.9 & 9.1 & 8.9 & 8.4 & 7.7
\end{tabular}

Fuente: División de Epidemiología H ospitalaria, IN N SZ sentaron $24.6 \%$. Dentro de las infecciones de herida quirúrgica, los gérmenes aislados más importantes fueron E. coli $(26.1 \%)$, Staphylococcus aureus (13.3\%), estafilococo coagulasa negativo (11.1\%), P. aeruginosa (9.1\%) y Enterobacter cloacae (8.7\%). Cabe mencionar que hubo un incremento estadísticamente significativo en la infección de herida quirúrgica por estafilococo coagulasa negativo ( $c^{2} 4.09$, RM 0.38, IC95\% 0.13-1.11, $p<0.5)$. En las neumonías nosocomiales, P. aeruginosa fue el patógeno predominante con $35.1 \%$ de los aislamientos, seguida en menor proporción por E. coli, Candida spp., estafilococo coagulasa negativo y E. cloacae.Se pudo observar una disminución estadísticamente significativa en la frecuencia de $P$. aeruginosa como causante de neumonía $\left(c^{2}\right.$ 4.8, RM2.89, IC95\% 1-8.58, p< 0.05). Delos gérmenes aislados para bacteriemia primaria, estafilococo coagulasa negativo se identificó en $19.6 \%$, seguido por estafilococo coagulasa positivo con $12 \%$, E. coli con $11.9 \%$, P. aeruginosa con $10.2 \%$ yE. cloacae con $8.5 \%$.

Cuadro II

RAZÓN DE INFECCIONESPOR 100 EGRESOS EN LOSSECTORESDE HOSPITAUZACIÓN DEL INNSZ, 1991-1996

\begin{tabular}{lrrrrrr} 
& 1991 & 1992 & 1993 & 1994 & 1995 & 1996 \\
& & & & & & \\
UTI* & 27.8 & 31.3 & 26.1 & 30.6 & 19.2 & 26.3 \\
\hline Piso 1 & 11.5 & 9.0 & 10.2 & 7.8 & 8.8 & 7.6 \\
\hline Piso 2 & 8.6 & 10.2 & 11.1 & 11.2 & 9.8 & 8.4 \\
\hline Piso 3 & 7.8 & 6.2 & 6.8 & 9.1 & 7.1 & 7.3 \\
\hline Urgencias & 12.9 & 7.4 & 11.1 & 5.4 & 7.1 & 2.3 \\
& \\
*Unidad de terapia intensiva
\end{tabular}

Fuente: División de Epidemiología Hospitalaria, IN N SZ

\section{Cuadro III \\ STIOSDE INFCCIÓN NOSOCOMIAL MÁSFRECUENTES EN EL IN NSZ, 1991-1996*}

\begin{tabular}{lrrrrrr} 
Vías urinarias & 27.2 & 24.1 & 29.7 & 31.3 & 25.7 & 21.5 \\
\hline Herida quirúrgica & 23.4 & 22.7 & 18.6 & 25.4 & 29.3 & 27.1 \\
\hline N eumonía & 9.3 & 10.2 & 10.5 & 11.4 & 12.3 & $18.9^{\ddagger}$ \\
\hline Bacteriemia primaria & 15.1 & 13.3 & 12.1 & 5.2 & 7.1 & 4.4 \\
\hline Bacteriemia secundaria & 4.6 & 5.2 & 4.9 & 8.1 & 4.8 & 3.1 \\
& \\
* Tasa porcentual \\
₹ Brote en hospitalización (1996)
\end{tabular}

Fuente: División de Epidemiología Hospitalaria, IN N SZ 
Aunque no se encontraron más diferencias significativas entre los microrganismos aislados, cabe observar que en algunosseaprecia una clara tendencia hacia un mayornúmero de aislamientos si se comparan 1991 y 1996. Así, por ejemplo, enterococo, como causante de infección de vías urinarias, se aislóen 1\% del total deepisodios en 1991, y para 1996 aumentó a 13\%; en neumonías nosocomiales producidas por Candida spp., en 1991 se aisló en $2.9 \%$ del total de los episodios, mientras que en 1996 fue en 10\%; en 1991 S. aureus se aisló en $16.3 \%$ de los episodios de bacteriemias primarias, con un decremento a $7.1 \%$ en 1996.

La mortalidad asociada a IN (mortalidad cruda en pacientes con IN) disminuyó en 36\% durante el periodo de estudio ( $\mathrm{c}^{2}$ 17.07, RM 2.52, IC95\% 1.58-4.07, $p<0.0001$ ) (figura 1). La edad se mantuvo en un promedio de 51.1 años en los diferentes periodos. Tampoco se encontró diferencia significativa en la gravedad de los pacientes atendidos entre 1991 y 1993, comparados con 1996 en los grupos con sobrevida mayor de seis meses $\left(c^{2}\right.$ 0.06 , RM1.02,IC95\% $0.87-1.8, p=0.8$ ) y el grupo con sobrevida menor de seis meses (rápidamente fatales: $\mathrm{c}^{2} 1.0, \mathrm{RM} 0.78$, IC95\% 0.47-1.31, $p=0.3$ ) (cuadro IV). En cambio, en el promedio de estancia hospitalaria en los pacientes con IN seobservó una disminución de $42.8 \%$ (de 40.6 días promedio en 1991 a 23.2 días en 1996) (cuadro IV).

\section{Discusión}

El objetivo primordial de un programa de control de infecciones es reducir la frecuencia, la morbilidad y la mortalidad asociadas a IN, por medio de la vigilancia continua, el mejoramiento de las condiciones de atención de los pacientes y la preparación del personal en materia de prevención y control de infecciones. El INNSZ tiene un programa de control de infecciones que funciona desde hace 11 años. Entre 1982 y 1984 la tasa de infecciones en el INNSZ fue de 19.5 por 100 egresos; ${ }^{10}$ para 1991 había disminuido a 12.5\%. Durante los siguientes cinco años, continuó disminuyendo hasta alcanzar una tasa de $10 \%$ en 1996; es decir, que durante esos años disminuyó en $20 \%$. Así entonces, a partir de la puesta en marcha de ese programa la tasa de infecciones en el INNSZ se ha reducido en $56 \%$, lo cual se traduce en una disminución anual aproximada de $4 \%$.

Como sucede en otros hospitales, la frecuencia de IN en la UTI es de dos a tres veces más elevada que en el resto del hospital. ${ }^{16-18}$ Llama la atención que, a diferencia de lo que ha ocurrido en la mayoría de las áreas del hospital, en la UTI la frecuencia de IN ha permanecido constante. Por otra parte, la disminución de las infecciones en otras áreas hospitalarias puede atribuirse a varios factores: a) a la reducción en el número de camas/cuarto durante el periodo estudiado (de seis camas en 1995 a cuatro camas por cuarto

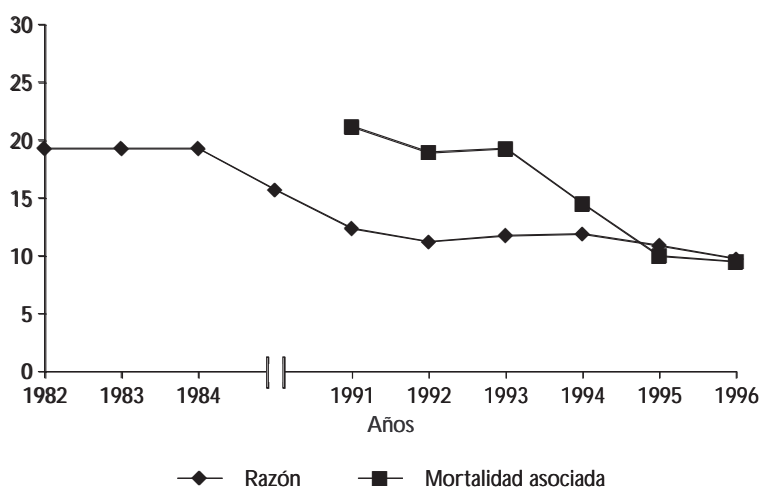

FigURA 1.TENDENCIASDE MORTALDAD ASOCIADA Y RAZÓN DE INFECCIONES NOSOCOMIALES EN EL INNSZ, 1982-1996

\section{CuadroIV \\ FACTORESASOCIADOSA INFECIONESNOSOCOMIALES EN EL IN NSZ, 1991-1996}

$\begin{array}{llllll}1991 & 1992 & 1993 & 1994 & 1995 & 1996\end{array}$

\begin{tabular}{lrrrrrr} 
Mortalidad asociada & 26.6 & 19.1 & 19.4 & 14.8 & 10.0 & 9.7 \\
\hline Estancia hospitalaria* $^{*}$ & 40.6 & 33.7 & 30.3 & 24.1 & 22.8 & 23.2 \\
\hline Promedio de edad $^{\ddagger}$ & 50.6 & 50.2 & 52.7 & 50.5 & 51.7 & 50.8 \\
\hline Rápidamente fatales $^{\S}$ & 5.3 & 5.1 & 12.2 & 9.9 & 3.3 & 9.8 \\
\hline Ultimamente fatales $^{\S}$ & 49.1 & 45.3 & 39.2 & 34.4 & 50.8 & 44.4 \\
\hline No fatales $^{\S}$ & 45.6 & 49.7 & 48.6 & 55.7 & 45.9 & 45.8
\end{tabular}

* Días

${ }^{\ddagger}$ Años

${ }^{\S}$ Escala de MacC abe-Jackson (gravedad de la enfermedad)

en 1996), aunque la disminución más importante ocurrió antes de dicho periodo; b) al programa de vigilancia y control de infecciones nosocomiales, ${ }^{8,10} \mathrm{y}$ c) a la disminución en la estancia hospitalaria en 42.8\% (40.6 días promedio en 1991 a 23.2 días en 1996) a lo largo de los cinco años que comprendió este estudio. .11,12,19,20 $^{2}$

Dicha disminución no puede explicarse por variaciones en el promedio de edad de los pacientes que fueron atendidos en el INNSZ durante el periodo de estudio, ni por la gravedad de las enfermedades subyacentes evaluadas por la escala de MacCabe-Jackson (cuadro IV). Las diferencias más importantes se encontraron en los pisos de hospitalización primero y segundo, donde ocurrieron los cambios antes mencionados, ya que en el tercer piso, donde 
había cubículos individuales, no se observaron cambios en la razón de IN durante ese periodo.

Entre 1991 y 1996 la frecuencia de infección de vías urinarias ha disminuido. Esa tendencia puede atribuirse a la supervisión de los procedimientos de colocación y a los cuidados de los sistemas de drenaje urinario cerrados ${ }^{21}$ así como a la capacitación continua del personal. Asimismo, las bacteriemias primarias disminuyeron en $70 \%$ durante los últimos cinco años (tasa de 15.1 en 1991 a 4.4 en 1996). Lo anterior es particularmente importante, ya que se ha reportado que la mortalidad asociada a bacteriemia es de $50 \%$ y la mortalidad atribuible en pacientes graves puede llegar hasta 35\%. ${ }^{22-24}$ La utilización de catéteres es un factor de riesgo para el desarrollo de bacteriemias, especialmente en áreas donde son muy requeridos. A partir de 1981 el Centro para la Prevención y el Control de Enfermedades de EUA (CDC) ha elaborado guías para el uso adecuado de catéteres, mismas que se han utilizado en el INNSZ; además, desde 1996 se dispone de un equipo de terapia intravenosa que ha permitido reducir en $50 \%$ la frecuencia de bacteriemias, en comparación con el año anterior. En cambio, las infecciones de herida quirúrgica y las neumonías han aumentado notablemente; cabe citar que en 1996 hubo un brote de neumonías en hospitalización que elevó aún más la frecuencia de estas infecciones.

En el INNSZ sigue siendo $E$. coli la causa más frecuente de infección de vías urinarias; sin embargo, Candida spp. le sigue en importancia y refleja indirectamente la gravedad de los pacientes que son atendidos en el INNSZ, dado que característicamente este microrganismo es un agente oportunista. Durante el periodo de estudio la frecuencia de aislamientos de enterococo por episodios de infecciones de vías urinarias se ha incrementado de 1\% en 1991, a 13\% en 1996. Estos últimos atraen un interés particular por su resistencia a antimicrobianos de amplio espectro. ${ }^{25} \mathrm{~A}$ diferencia de lo informado por el sistema de vigilancia de infecciones nosocomiales del CDC, en el INNSZ los gramnegativos siguen siendo la causa más frecuente de infecciones quirúrgicas, seguidos por estafilococo coagulasa negativo, el cual ha aumentado significativamente. En las infecciones de herida posquirúrgica, E. coli y los cocos grampositivos fueron los predominantes; de estos últimos, el estafilococo coagulasa negativo ha aumentado en los últimos años. ${ }^{26}$

Las IN son responsables de un aumento tanto en la mortalidad asociada, como en el número de días de estancia hospitalaria de los pacientes que las padecen. ${ }^{8,12,17}$ En este estudio se pudo observar una disminución importante de la mortalidad asociada, demostrando además que dicha reducción no está re- lacionada con el hecho de que se internen pacientes menos graves pues, como ya se mencionó, no hubo diferencias en la tendencia del estado de gravedad de los pacientes egresados en todo ese periodo. Por otra parte, se revisó el número de altas voluntarias, pues muy a menudo ese tipo de altas son pacientes desahuciados que no fallecen en el hospital. Durante el lapso en cuestión tampoco se encontraron diferencias estadísticamente significativas $\left(\mathrm{c}^{2}\right.$ 1.77, RM0.84, IC95\% 0.65, $1.09 ; p=0.18$ ).

En 1985 Haley y colaboradores demostraron que la puesta en marcha de un programa de vigilancia y control de IN, formado por un médico entrenado, una enfermera por cada 250 camas y un sistema de reporte de infecciones quirúrgicas, disminuyó la frecuencia de las IN en $32 \%{ }^{8}$ En el INNSZ se ha logrado una disminución de $56 \%$ a partir del momento en que arrancó el programa de control de infecciones nosocomiales, cifra que confirma su eficacia y trascendencia en el plano de la calidad de la atención hospitalaria.

El aspecto que reviste mayor interés en este estudio es que la mortalidad asociada a IN se redujo en $36 \%$, hecho no publicado previamente como secundario a un programa global de vigilancia y control de infecciones nosocomiales. Si bien se evaluaron la gravedad de la enfermedad subyacente y la edad de los pacientes, en estudios posteriores podría controlarse no sólo la gravedad sino también el tipo de enfermedad subyacente y otras comorbilidades que permitan conocer el verdadero impacto que tienen estos programas en la mortalidad atribuible. De cualquier manera, esta observación debe agregarse a los muchos beneficios que estos programas tienen en los hospitales. Por otra parte, si bien la aplicación de medidas específicas para controlar las infecciones permite reducir su frecuencia, tal como se ha demostrado aquí, la ocurrencia de infecciones se mantendrá en un mínimo irreductible, consecuencia de las características inherentes a las condiciones en que se atiende a los pacientes en el ámbito hospitalario. ${ }^{6}$

\section{Referencias}

1. Haley RW, Culver DH, W hite JW, Morgan W M, Emori TG. The nationwide nosocomial infection rate:A new need for vital statistics. Am J Epidemiol 1985;121:159-167.

2. Ponce de León $\mathrm{S}$. The needs of developing countries and the sources required.J Hosp Infect 1991;18 suppl A:376-381.

3. O rganización Panamericna de la Salud-0 rganización Mundial de la Salud. Directorio Latinoamericano y del Caribe de Hospitales.W ashington, D.C.: O PS-O MS, 1995.

4. Ponce de León S, Rangel S. O rganizing for infection control with limited resources. En:W enzel RP, ed. Prevention and control of nosocomial infections. Baltimore:W illiams \& W ilkins, 1993:82-88.

5.Young LS. N osocomial infection in inmunocompromised adult.Am J Med 1981;70:398-404.

6. Ayliffe GA, FRCPath. N osocomial infection:The irreductible minimum. Infect Control 1986;7 suppl 2:92-95. 
7.N ettleman M.The global impact of infection control.En:W enzel RP,ed.Prevention and control of nosocomial infection.2a.edición. Baltimore:W illiams \&W ilkins,1993:13-20. 8. Haley RW, Culver DH,W hite JW.The efficacy of infection surveillance and control programs in preventingnosocomial infection in UShospitalAmJ Epidemio 1985;121:182205.

9. W enzel RP. N osocomial infection: Diagnosis-related groups and study on the efficacy of nosocomial control. Am J Med 1985;78 suppl 6B:3-7.

10. Ponce de León S, Romero M, Sandoval M, Ruiz G Eficacia de un programa de control de infecciones nosocomiales: una posibilidad real para mejorar la calidad de la atención médica. Salud Publica Mex 1986;28:593-598.

11. Green JW,W enzel RP.A controlled study of the increased duration of hospital stay and direct costs of hospitalization. Ann Surg 1977;185: 264-268.

12. Haley RW, Schaberg D, Crossley KB, Von Allimen SD, McGowan JE. Extracharges and prolongation of stay attributable to nosocomial infection:A prospective interhospital comparison. Am J Med 1981;70:51-58.

13. Britt MZ, Schleupner CJ, MatsumigaS. Severity of underlyingdisease as a predictor of nosocomial infection. Utility in the control of nosocomial infection.JAMA 1978;239:10471051.

14. Morales GD, Ponce de León RS, Rangel-Frausto MS.Validación de un programa de vigilancia de infecciones nosocomiales. Enfermedades Infecciosas y Microbilogía 1995;15:318.

15.Vincent JL, Bihari DJ, Suter PM.The prevalence of nosocomial infection in intensive care unit in Europe. JAMA 1995;274:639-644.

16. Bueno-C anavillas A, D elgado-Rodríguez M, López-Luque A, Schaffino$C$ ano S, Gálvez-Vargas R. Influence of nosocomial infection on mortality rate in an intensive care unit. C rit C are Med 1994;22(1):55-60.

17. Fagon J, Novara A, Stephan F. Mortality attributable to nosocomial infection in the ICU. Infect Control Hosp Epidemiol 1994;15:428-434.

18. McC abe W R, Jackson G G. Gram-negative bacteremia: I. Etiology and ecology.Arch Intern Med 1962;110:847-864.

19.W ey SB, Mori M, Pfaller MA, W enzel RP. Hospital adquired candidemia: The attributable mortality and excess length of stay. Arch Intern Med 1988;148:2642-2647.
20.Martin MA,Pfaller MA,W enzel RP.C oagulase-negative staphylococcal bacteremia: Mortality and hospital stayAnn Intern Med 1989;110:9-16.

21. Burke JP, Larsen RA, Steven LE. Estimating the potential for prevention by closed sterile urinary drainage. Infect Control Hosp Epidemiol 1986;7 suppl 2:96-99.

22. Pittet $D$, Tarara $D$, W enzel RP. N osocomial bloodstream infection in critical III patients. JAMA 1994;271:1598-1601.

23. Pittet D. N osocomial bloodstream infections. En:W enzel RP, ed. Prevention and control of nosocomial infections. 2a. edición. Baltimore:W illiams \& W ilkins, 1993:512-555

24. W enzel RP.The mortality of hospital-acquired bloodstream infection: N eed for a new vital statistic? Int J Epidemiol 1988;17:225.

25. García-García L. Infecciones urinarias intrahospitalarias. En: Ponce de León-Rosales S, ed. Infecciones Intrahospitalarias. México, D.F.: McG rawHill Interamericana, 1996:87-95.

26. Mayhall C. Surgical infections including burns. En:W enzel RP, ed. Prevention and control of nosocomial infections. 2a. edición. Baltimore: W illiam \& W ilkins, 1993:614-664. 